\title{
Ileo-sigmoid knotting: the Parirenyatwa hospital experience
}

\author{
C Mbanje, ${ }^{1}$ SG Mungazi, ${ }^{3}$ D D Muchuweti, ${ }^{1}$ D Mazingi, ${ }^{1}$ M Mlotshwa, ${ }^{2}$ AJV Maunganidze ${ }^{1}$ \\ ${ }^{1}$ Department of Surgery, College of Health Sciences, University of Zimbabwe, Zimbabwe \\ ${ }^{2}$ Colorectal Surgery, Western Sussex Hospitals, National Health Services Trust, United Kingdom \\ ${ }^{3}$ Department of Surgery and Anaesthetics, Faculty of Medicine, National University of Science and Technology, Zimbabwe
}

Corresponding author, email: sgmungazi@gmail.com

Background: Ileo-sigmoid knotting is a rare cause of intestinal obstruction with a rapidly progressive course, for which expedient surgical intervention is required to prevent mortality. The aim of this study was to determine the characteristics, presentation, morbidity and mortality associated with ileo-sigmoid knotting at Parirenyatwa Group of Hospitals (PGH). To determine the preoperative diagnostic precision and management patterns of ileo-sigmoid knotting cases at PGH.

Methods: A retrospective analysis was performed on patients operated on at Parirenyatwa Hospital with a diagnosis of ileo-sigmoid knotting between April 2011 and April 2018. Data inclusive of demographics, time to presentation and surgery, preoperative diagnosis, complications and in-hospital mortality was collected. The relationship between the duration of symptoms prior to surgery and incidence of both septic shock and transfusion were analysed.

Results: Twenty-one cases of ileo-sigmoid knotting were identified for analysis. The median age was 37 years (range 18-65 years) with a 6:1 male to female ratio. Two of the three females included were pregnant. Twenty patients (95.2\%) described an acute onset abdominal pain, with $83.3 \%$ experiencing the pain nocturnally, while asleep. The median duration of symptoms at presentation was 12.5 hours (range $2-39$ hours). At admission, leucocytosis (WCC $>11 \times 10^{3} / \mathrm{dl}$ ) was noted in eleven patients $(52.4 \%)$. Seventy-three per cent of patients were noted to have electrolyte derangements at presentation. Seven patients $(33.3 \%)$ had recorded episodes of severe hypotension (SBP $<90)$ prior to surgery. The most common preoperative diagnosis, based on both clinical assessment and plain x-ray evaluation, was sigmoid volvulus $(52.4 \%)$, with no preoperative diagnosis of ileo-sigmoid knotting being made. All patients had gangrenous small bowel, with $81 \%$ having a gangrenous sigmoid colon. All cases underwent small bowel resection and primary anastomosis plus Hartmann's procedure. Postoperatively, eleven patients (52.4\%) developed septic shock, while $62 \%$ required blood transfusion. There was one $(4.8 \%)$ early postoperative mortality.

Conclusion: To avoid mortality, the diagnosis of ileo-sigmoid knotting should be entertained and the imperative of emergency surgery recognised in the young male or pregnant female patient with acute nocturnal onset abdominal pain, a rapidly deteriorating small bowel obstruction clinical picture and with radiological features suggestive of both small and large bowel obstruction.

Keywords: ileo-sigmoid knot, compound volvulus, intestinal obstruction, volvulus

\section{Introduction}

Ileo-sigmoid knotting (ISK), (also referred to as double volvulus or compound volvulus), is an unusual and rare cause of intestinal obstruction in which small bowel or sigmoid colon wrap around each other, causing double loop obstruction and frequently leading to bowel strangulation. ${ }^{1-3}$ The condition generally progresses rapidly to strangulation, ischaemia and gangrene of both loops of bowel and eventual death, if emergent surgery is not undertaken. Preoperative diagnosis is rare in the absence of advanced imaging, which is often not available in areas where this condition is endemic. ${ }^{4}$ The limited ability for early diagnosis and intervention is said to be associated with the high mortality that has been reported to range between $20-100 \%$ in the presence of gangrene. ${ }^{1,4-6}$

Ileo-sigmoid knotting is a rare cause of intestinal obstruction in the West, although it is a recognised cause of intestinal obstruction in parts of Africa, Asia, and the Middle East with a high prevalence of sigmoid volvulus. , $^{1,-6}$ The reasons are largely unknown but may be related to anatomical and dietary peculiarities in these populations. Peak incidence has been noted to occur in the third to fifth decades of life, with a strong male predominance. It is generally uncommon in females, and when it does occur, it is usually in the setting of pregnancy. ${ }^{7}$

The largest reported series in the literature is that of Shepherd, in Uganda, with 92 cases in 1967. ${ }^{1}$ More recently in 2013, Atamanalp presented a retrospective review of 73 cases in Turkey. ${ }^{4}$ The aim of this study was to analyse the clinical presentation, management patterns and outcomes of ISK in Zimbabwe at Parirenyatwa Group of Hospitals $(\mathrm{PGH})$ around Harare and to analyse factors which may influence outcome. 


\section{Methodology}

A retrospective review of all cases of surgically determined ISK at PGH over the period April 2011 to April 2018 was performed. Sources included patient medical records as well as interview of patients or the attending surgeon where these were available. Data was collected and analysed in a Microsoft Excel spreadsheet, capturing demographics, onset and duration of symptoms prior to presentation and subsequent surgery, preoperative diagnostic accuracy, degree of decompensation at surgery, surgical management, as well as final patient outcomes. The relationship between total symptom duration prior to surgery and the incidence of septic shock and anaemia requiring transfusion were also analysed.

\section{Results}

Twenty-four patients had surgery for ISK between April 2011 and April 2018. Three patients were excluded due to incomplete record leaving 21 patients available for analysis. There was a male to female ratio of 6:1. The median age was 37 years (range 18-65), with a modal incidence in

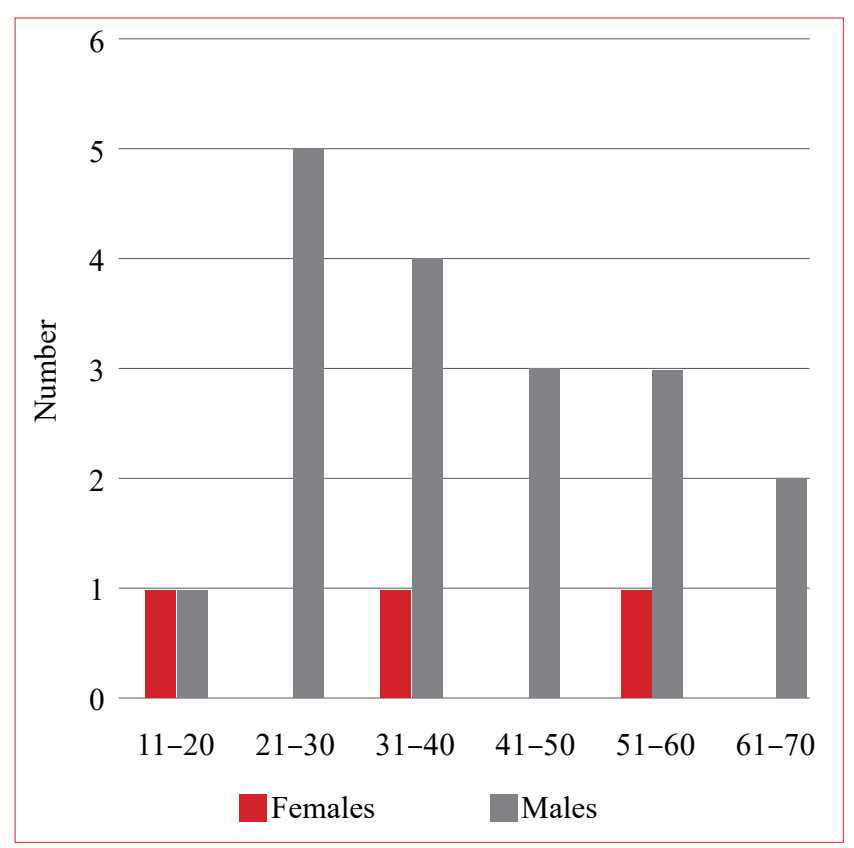

Figure 1: Age and sex distribution



Figure 2: A radiological picture of mixed large and small bowel dilatation was seen in most radiographs. A common feature was a prominent dilated large bowel loop mimicking sigmoid volvulus but of smaller dimensions compared to the typical "omega" loop. No patients obtained preoperative computerised tomography due to unavailability the third and fourth decades $(53.3 \%)$. Two (2) of the three female patients, were in their thirteenth week of pregnancy (Figure 1).

Twenty patients $(95.2 \%)$ described an acute onset of rapidly progressive severe abdominal pain necessitating immediate hospital transfer. Eighty-three per cent were able to relate that the onset of pain woke them up from sleep, while $17 \%$ described the pain as commencing in the "early morning". The median time from onset of symptoms to definitive surgery was 27.2 hours (range 15-61 hours). The median time from presentation at hospital to surgery was 12.5 hours (range $4.5-59$ hours).

Seven patients $(33.3 \%)$ had recorded episodes of severe hypotension ( $\mathrm{SBP}<90 \mathrm{mmHg}$ ) prior to surgery. At admission, leucocytosis (WCC $>11 \times 10^{3} / \mathrm{dl}$ ) was noted in eleven patients $(52.4 \%)$, while haemoglobin levels ranged from $10.0-17.6 \mathrm{~g} / \mathrm{dl}$, with a median of $14.6 \mathrm{~g} / \mathrm{dl}$. Seventythree per cent of patients were noted to have electrolyte derangements at presentation, inclusive of hyperkalaemia (K $>5.0 \mathrm{mEq} / \mathrm{l}$ ) and raised urea (urea $>7 \mathrm{mmol} / \mathrm{l}$ ). These results did not take into account the possibility of any underlying comorbidities.

No clinical or radiological diagnosis of ISK was made preoperatively. The commonest preoperative diagnosis was sigmoid volvulus in 11 cases $(52.4 \%)$, followed by ruptured appendix $(23.8 \%)$. Other diagnoses included perforated ulcer, pancreatitis and ectopic pregnancy. Peritonitis was the predominant reason for taking patients for laparotomy. All patients had preoperative plain abdominal radiographs. The plain films shown in Figure 2 illustrate the variability of findings.

\section{Management}

At laparotomy, all cases had small bowel gangrene, with an $81 \%(17 / 21)$ incidence of sigmoid colon gangrene (Figure 3). All cases were type 1 knots as described by Alver, with small bowel as the active component. ${ }^{5}$ Gangrenous small bowel was resected with primary handsewn end-toend anastomoses with absorbable sutures. Sigmoid resection and Hartmann's procedure were performed in all cases regardless of the presence or absence of colonic gangrene.

All patients were admitted in the intensive care unit postoperatively as per standing unit protocol and discharged to lower care wards as condition permitted. Inotropic support was commenced either intraoperatively or postoperatively as required.

\section{Outcomes}

\section{Complications}

Eleven patients $(52.4 \%)$ were commenced on inotropic support perioperatively as a result of bowel gangreneinduced septic shock. Sixty-two per cent experienced a significant drop in haemoglobin postoperatively requiring transfusion of packed cells, with a transfusion trigger of $8.1 \mathrm{~g} / \mathrm{dl}$. Respiratory complications (inclusive of pleural effusion, respiratory distress syndrome) were noted in $28.6 \%$ of patients. There was one anastomotic leak (4.8\%).

When stratified according to whether patients were operated on less than or more than 24 hours after symptom onset, there were obvious disparities in incidence of both septic shock and transfusion requirement as illustrated in Figure 4. 


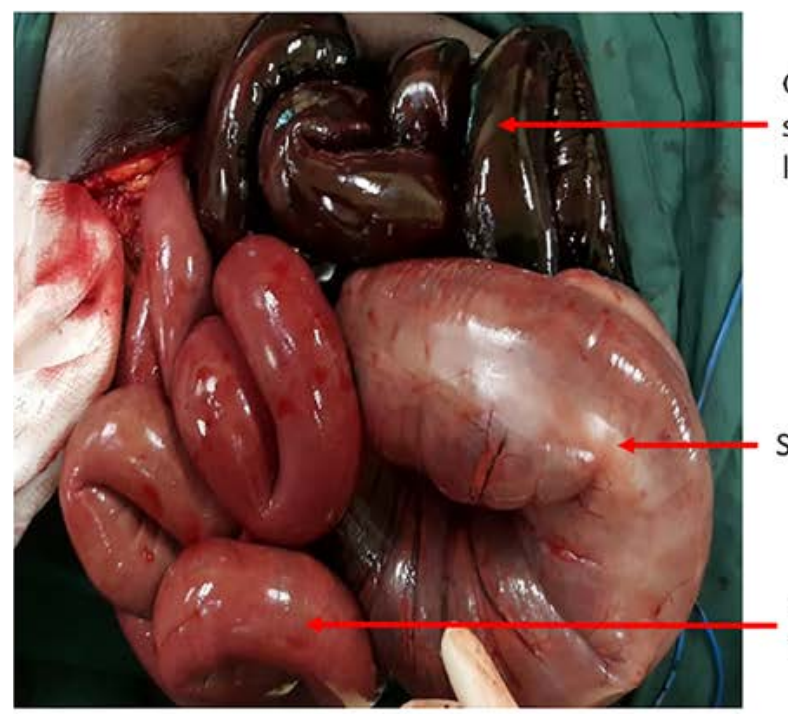

Gangrenous

small bowel

loops

Sigmoid colon

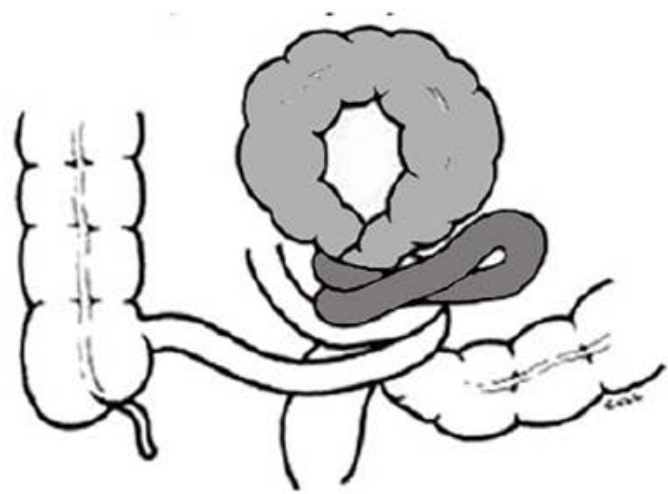

Figure 3: Intraoperative images illustrating findings at laparotomy. Gangrene was present in both sigmoid colon and small bowel. On the right, the cartoon depicts events occurring on the left, with small bowel tightly wrapped around the base of the sigmoid colon and causing strangulation of both sigmoid colon and small bowel loops distal to twist (image adapted from Mandal et al. ${ }^{8}$ )

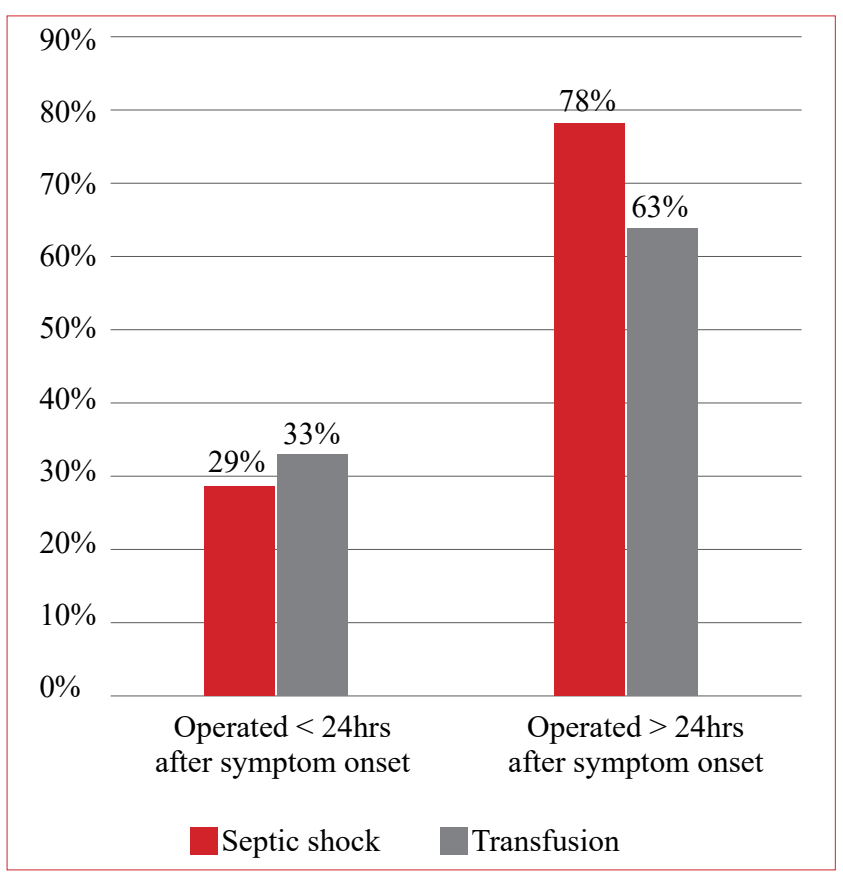

Figure 4: Incidence of septic shock and transfusion based on duration of symptoms. There was a statistically significant difference in incidence of septic shock $(p=0.02)$ but not transfusion requirement $(p=0.16)$

\section{Length of stay and mortality}

The median length of hospital stay was 12 days (range 5-18 days) The median length of critical care unit stay was 3 days (range 1-8 days). There was one death in the early postoperative period, giving an in-hospital mortality rate of $4.8 \%$.

\section{Discussion}

ISK is a relatively uncommon cause of the acute abdomen. The condition is frequently reported in areas with a high incidence of sigmoid volvulus. This may be linked to similarities in the patho-anatomy of the sigmoid colon common to both conditions, i.e. the dolichosigmoid. ${ }^{6,9,10}$ Indeed, Alver et al. reported a series in which $26.5 \%$ of patients presenting with ISK had reported prior episodes of sigmoid volvulus. ${ }^{5}$ Unlike sigmoid volvulus, however, this condition almost invariably takes on a precipitous course, requiring emergency surgery. Other anatomical factors, such as an elongated small bowel mesentery and laxity of the abdominal wall as occurs in pregnancy or deep sleep, have been linked with this condition. ${ }^{3,5,6}$ While an increasing number of cases are reported in literature, these are mostly retrospective case reports, thus our knowledge of the evolution of this condition remains limited.

In most instances, the diagnosis is made at laparotomy as there are no recognised pathognomonic symptoms, signs, or radiological features to allow for a definitive preoperative diagnosis., ${ }^{4,11,12}$ The combination of clinical features of small bowel obstruction with radiological features of large bowel obstruction have been described as contradictory and contributing to diagnostic uncertainty. ${ }^{13}$ ISK is endemic to areas that are of a lower socio-economic setting, and as such, use of specialised imaging (e.g. CT scans) in the emergency setting that could assist in establishing an early definitive diagnosis is limited. Early recognition of this pathological process is important in mitigating against unnecessary delays to definitive surgery, as may occur when the diagnosis is uncertain.

Our results illustrated a higher incidence in adult males, in keeping with previously published literature..$^{4,5,11,12}$ It has also been noted that when the condition occurs in females, multiparity and late stage pregnancy are identified as potential contributory factors owing to the increased laxity of the abdominal wall musculature from hormonal influences as well as mass effect. ${ }^{7}$ However, it is of interest that in our study population, two of the three female patients were in their first trimester of pregnancy, suggesting that there may be as yet unexplained influences of pregnancy contributing to the pathogenesis of ISK.

Of particular interest, ninety-three per cent of our patients described an acute onset of severe abdominal pain, with $83.3 \%$ describing the pain as being of nocturnal onset 
and awakening the patient from sleep. This is in keeping with the postulated pathophysiology, in which laxity of the abdominal musculature as occurs during deep sleep, in combination with anatomical variations, allows bowel movements necessary for knot formation. ${ }^{2,6}$ Considering the frequency of this finding in our series, the timing of the onset of pain is an important, frequently neglected, point of note in the diagnostic evaluation.

The most common preoperative diagnosis, similar to findings in other series, was sigmoid volvulus $(52.6 \%))^{4,5}$ This was largely supported by plain radiographic findings of combined small and large bowel dilatation, but with a prominent large bowel loop that mimicked but was of smaller calibre than the typical 'omega loop' of the more common sigmoid volvulus. Other radiological features previously described, e.g. medial deviation of the distal descending colon or soft tissue shadowing in the left iliac fossa, were not consistently noted in general and in this series. ${ }^{14}$

Raveenthiran described a diagnostic triad of clinical clues in an attempt to enable greater preoperative diagnostic accuracy. ${ }^{15}$ Two of these were similarly identified in our patients, namely a clinical picture of small bowel obstruction and radiological evidence of large bowel obstruction. The third, failure to pass a sigmoidoscope, was not attempted in our patients and was considered in retrospect to be potentially harmful in view of the high incidence of bowel gangrene. An alternative clinical symptom, that of nocturnal onset severe abdominal pain, was noted with high frequency in our patient population. Being a non-invasive clinical clue, it is suggested as an alternative component of a predictive triad.

Most of our patients presented within 24 hours of symptom onset, which was relatively early compared to older series, but similar to recent findings by Ooko et al., ${ }^{4,5,11,12}$ In spite of this, a large proportion of patients had evidence of significant physiological derangements at admission and gangrenous bowel at surgery, highlighting the precipitous pathological course. Interestingly, previous studies have not conclusively linked delays to definitive surgery with poorer outcomes as expected..$^{4,5,12,15}$ In our series, there were significant disparities in the incidence of septic shock based on time to definitive surgery (Figure 4 ). Given that $94 \%$ of patients in this series presented to hospital within 24 hours of symptom onset, but only $33 \%$ were operated within 24 hours, it is possible that in-hospital delays in diagnosis and treatment contributed to increased patient morbidity. Considering the rapidly progressive course of this disease entity, it is likely that patients who experienced extended delays (both pre-hospital and in-hospital), as is common in low Human Development Index countries, are likely to have demised before surgery could be undertaken and a definitive diagnosis made. The comparatively favourable mortality rate of $4.8 \%$ in our cohort may therefore be a result of a combination of factors, inclusive of a relatively early time to care, mandatory postoperative ITU stay, improved critical care as compared to earlier series as well as the avoidance of morbidity related to emergent left-sided colonic anastomosis in these patients. ${ }^{1,4,5,13}$

\section{Conclusion}

In the management of ISK, early intervention is vital to improved outcomes. In the young adult male, or pregnant female patient presenting with an acute abdomen, the presence of the triad of acute nocturnal onset severe abdominal pain, a rapidly deteriorating small bowel obstruction clinical picture and radiological features suggestive of small and large bowel obstruction, the diagnosis of ISK should be entertained and the imperative of emergency surgery must be recognised.

\section{Conflict of interest}

The authors declare no conflict of interest.

\section{Funding source}

No funding was required.

\section{Ethical approval}

Ethics approval was obtained from the Joint Research Ethics Committee of the University of Zimbabwe, JREC Ref: 202/18.

\section{ORCID}

SG Mungazi (D) https://orcid.org/0000-0001-8048-4866

D Mazingi (D) https://orcid.org/0000-0001-8217-4642

\section{REFERENCES}

1. Shepherd JJ. Ninety-two cases of ileosigmoid knotting in Uganda. Br J Surg. 1967 Jun;54(6):561-6.

2. Vaez-Zadeh K, Dutz W. Ileosigmoid knotting. Ann Surg. 1970 Dec;172(6):1027-33.

3. Atamanalp SS. Ileosigmoid knotting. Eurasian J Med. 2009 Aug;41(2):116-9.

4. Atamanalp SS. Ileosigmoid knotting: clinical appearance of 73 cases over 45.5 years. ANZ J Surg. 2013;83(1-2):70-3.

5. Alver O, Oren D, Tireli M, Kayabaşi B, Akdemir D. Ileosigmoid knotting in Turkey. Review of 68 cases. Dis Colon Rectum. 1993 Dec;36(12):1139-47.

6. Mallick IH, Winslet MC. Ileosigmoid knotting. Colorectal Dis. 2004;6(4):220-5.

7. Atamanalp SS. Ileo-sigmoid knotting in pregnancy. Turk $\mathbf{J}$ Med Sci. 2012;42(4).

8. Mandal A, Chandel V, Baig S. Ileosigmoid knot. Indian J Surg. 2012 Apr;74(2):136-42.

9. Madiba TE, Haffajee MR. Sigmoid colon morphology in the population groups of Durban, South Africa, with special reference to sigmoid volvulus. Clin Anat NYN. 2011 May;24(4):441-53.

10. Madiba TE, Aldous C, Haffajee MR. The morphology of the foetal sigmoid colon in the African population: a possible predisposition to sigmoid volvulus. Colorectal Dis. 2015 Dec;17(12):1114-20.

11. Banerjee C, Mukhopadhyay M, Roy A, et al. The unusual volvulus: a five-year retrospective analysis of nine cases. Indian J Surg. 2014 Apr;76(2):100-3.

12. Ooko PB, Saruni S, Oloo M, Topazian HM, White R. Ileosigmoid knotting: a review of 61 cases in Kenya. Pan Afr Med J. 2016 Apr 15;23:198.

13. Machado NO. Ileosigmoid knot: a case report and literature review of 280 cases. Ann Saudi Med. 2009;29(5):402-6.

14. Young WS, White A, Grave GF. The radiology of ileosigmoid knot. Clin Radiol. 1978 Mar;29(2):211-6.

15. Raveenthiran V. The ileosigmoid knot: New observations and changing trends. Dis Colon Rectum. 2001 Aug 1;44(8):1196200 . 